\title{
Weaker D-Complete Logics
}

NORMAN D. MEGILL, 19 Locke Lane, Lexington, MA 02173, USA.

E-mail:ndm@shore.net

MARTIN W. BUNDER, Department of Mathematics, University of

Wollongong, Wollongong NSW 2500, Australia.

E-mail: Martin_Bunder@uow.edu.au

\begin{abstract}
$\mathbf{B B}^{\prime} \mathbf{I W}$ logic (or $T_{\rightarrow}$ ) is known to be $\mathbf{D}$-complete. This paper shows that there are infinitely many weaker $\mathbf{D}$-complete logics and it also examines how certain $\mathbf{D}$-incomplete logics can be made complete by altering their axioms using simple substitutions.

Keywords: condensed detachment
\end{abstract}

\section{Introduction}

The condensed detachment rule, first proposed by C. A. Meredith in Lemmon et al [5, is a form of modus ponens preceded by 'just enough' substitution to make the modus ponens possible. The substitution mechanism, for implicational formulas, was a precursor to Robinson's unification algorithm 8 .

Roughly, a system of implicational logic is $\mathbf{D}$-complete if the system with the same axioms, but with condensed detachment (D) instead of modus ponens and substitution, has the same theorems.

To show that a logic is $\mathbf{D}$-complete it is sufficient to show that all the substitution instances of its axioms are deducible in the corresponding condensed logic (i.e. the logic with rule $\mathbf{D}$ only).

It is well known that every logic with axioms only from the list:

(B) $\quad(a \rightarrow b) \rightarrow(c \rightarrow a) \rightarrow c \rightarrow b$

(B') $\quad(a \rightarrow b) \rightarrow(b \rightarrow c) \rightarrow a \rightarrow c$

(C) $\quad(a \rightarrow b \rightarrow c) \rightarrow b \rightarrow a \rightarrow c$

(K) $\quad a \rightarrow b \rightarrow a$

is $\mathbf{D}$-incomplete. (See Hindley and Meredith [3] and Kalman [4].)

Meyer and Bunder [6] showed that the system based on (B), (B'), (I) and

$(\mathrm{W}) \quad(a \rightarrow a \rightarrow b) \rightarrow a \rightarrow b$,

which we will call $\mathbf{B B}^{\prime} \mathbf{I} \mathbf{W}$ logic (or $T_{\rightarrow}$ ), is $\mathbf{D}$-complete. (See also [2] and 7]).

Here we show that $\mathbf{D}$-completeness can be shown for a weaker logic, which we will call $M$. This is based on (I) and the following:

$$
\begin{gathered}
(a \rightarrow a) \rightarrow(c \rightarrow b \rightarrow b) \rightarrow c \rightarrow(a \rightarrow b) \rightarrow a \rightarrow b \\
(b \rightarrow b) \rightarrow(c \rightarrow a \rightarrow a) \rightarrow c \rightarrow(a \rightarrow b) \rightarrow a \rightarrow b \\
(c \rightarrow a \rightarrow a) \rightarrow(c \rightarrow b \rightarrow b) \rightarrow c \rightarrow(a \rightarrow b) \rightarrow a \rightarrow b
\end{gathered}
$$


We show that there is an even weaker interesting $\mathbf{D}$-complete logic as well as an infinite sequence of independent $\mathbf{D}$-complete logics. We also comment on the relation between (A1), (A2) and (A3) and the more standard axioms such as (B), (B'), (I) and $(\mathrm{W})$.

\section{Condensed detachment}

The formulation we give here for condensed detachment is equivalent to the more standard one of, for example, Hindley and Meredith [3] but is simpler to state (see [1]).

In all the work below, if $P$ is a formula of $\operatorname{logic}, \sigma_{i}(P)$ will represent the result of a simultaneous substitution of formulas for propositional variables in $P$.

(Rule D). From $P \rightarrow Q$ and $R$ conclude $\sigma_{1}(Q)$, where there are substitutions $\sigma_{1}$ and $\sigma_{2}$ such that

(1) $\sigma_{1}(P)=\sigma_{2}(R)$.

(2) Given (1) the number of occurrences of propositional variables in $\sigma_{1}(Q)$ is minimal.

(3) Given (1) and (2), the number of distinct propositional variables in $\sigma_{1}(Q)$ is maximal.

Note 1. There may be no such $\sigma_{1}$ and $\sigma_{2}$, for example for $(a \rightarrow a) \rightarrow a \rightarrow a$ and $a \rightarrow b \rightarrow a$, so the above rule does not always reach a conclusion.

Note 2 . When we can obtain $\sigma_{1}(Q)$ from $P \rightarrow Q$ and $R$, we will say that we detach $R$ from $P \rightarrow Q$ to give $\sigma_{1}(Q)$.

We should also note that the axioms with names (I), (B) etc. are the principal types of the combinators $\mathbf{I}, \mathbf{B}$, etc. If a combinator $X$ has principal type $P \rightarrow Q$ and $Y$ has principal type $R$ then $X Y$, if it has a type, has principal type $\sigma_{1}(Q)$ obtained by $\mathbf{D}$. Thus $X Y$ can designate the proof of $\sigma_{1}(Q)$.

\section{The D-completeness of $M$}

The notation $\vdash_{M} P$ or just $\vdash P$ will represent " $P$ is a theorem of $M$."

Before proving our result we require 6 lemmas.

LEMMA 3.1

$\vdash(a \rightarrow a) \rightarrow(a \rightarrow a) \rightarrow a \rightarrow a$.

Proof. Detach (I) from (A3) to obtain:

$$
((a \rightarrow a) \rightarrow b \rightarrow b) \rightarrow(a \rightarrow a) \rightarrow(a \rightarrow b) \rightarrow a \rightarrow b .
$$

Detaching (I) from this gives the result.

Lemma 3.2

$\vdash(a \rightarrow a) \rightarrow a \rightarrow a$.

Proof. Detach (I) from lemma 3.1.

LEMMA 3.3

If a formula $P$ has no repeated propositional variables then

$$
\vdash P \rightarrow P .
$$


Proof. By induction on the length of $P$.

If $P$ is atomic, use (I).

If $P$ is $Q \rightarrow R$, where we have proved $\vdash Q \rightarrow Q$ and $\vdash R \rightarrow R$ in $M$, we detach $Q \rightarrow Q$ from (A1) to give

$$
\vdash(c \rightarrow b \rightarrow b) \rightarrow c \rightarrow(Q \rightarrow b) \rightarrow Q \rightarrow b .
$$

Detach lemma 3.2 from this to obtain

$$
\vdash(b \rightarrow b) \rightarrow(Q \rightarrow b) \rightarrow Q \rightarrow b .
$$

Detaching $R \rightarrow R$ from this gives

$$
\vdash(Q \rightarrow R) \rightarrow Q \rightarrow R
$$

as required.

Note that if $Q$ and $R$ had a variable in common, condensed detachment would have, at this last step, changed all occurrences of this common variable in $Q$ to a distinct new variable.

LEMMA 3.4

If a formula $P$ has no repeated propositional variables and $d$ is a propositional variable in $P$, then

$$
\vdash(d \rightarrow d) \rightarrow P \rightarrow P .
$$

Proof. By induction on the length of $P$.

If $P$ is atomic, use lemma 3.2 .

If $P$ is $Q \rightarrow R$, we have that $d$ is in $Q$ or $R$ but not both.

Case 1: $d$ is in $Q$. By the induction hypothesis

$$
\vdash(d \rightarrow d) \rightarrow Q \rightarrow Q
$$

and by lemma 3.3, $\vdash R \rightarrow R$.

Detach $R \rightarrow R$ from (A2) to obtain

$$
\vdash(c \rightarrow a \rightarrow a) \rightarrow c \rightarrow(a \rightarrow R) \rightarrow a \rightarrow R .
$$

Now detach $(d \rightarrow d) \rightarrow Q \rightarrow Q$ to give

$$
\vdash(d \rightarrow d) \rightarrow(Q \rightarrow R) \rightarrow Q \rightarrow R .
$$

Case 2: $d$ is in $R$. By the induction hypothesis

$$
\vdash(d \rightarrow d) \rightarrow R \rightarrow R
$$

and by lemma 3.3, $\vdash Q \rightarrow Q$.

Detach $Q \rightarrow Q$ from (A1) to obtain

$$
\vdash(c \rightarrow b \rightarrow b) \rightarrow c \rightarrow(Q \rightarrow b) \rightarrow Q \rightarrow b .
$$

Detach $(d \rightarrow d) \rightarrow R \rightarrow R$ to give

$$
\vdash(d \rightarrow d) \rightarrow(Q \rightarrow R) \rightarrow Q \rightarrow R
$$


LEMMA 3.5

Suppose $P$ is a formula with propositional variables $a_{1}, a_{2}, \ldots, a_{n}$ each of which appears exactly once in $P$. For $1 \leq i\langle j \leq n$ let

$$
Q=\left[a_{i} / a_{j}\right] P .
$$

Then $\vdash Q \rightarrow Q$.

Proof. By definition $a_{i}$ appears twice in $Q$. Let $R \rightarrow S$ be the largest part of $Q$ where $a_{i}$ appears in $R$ and in $S$.

As $a_{i}$ appears once in $R$ and once in $S$, we have by lemma 3.4

$$
\vdash\left(a_{i} \rightarrow a_{i}\right) \rightarrow R \rightarrow R
$$

and

$$
\vdash\left(a_{i} \rightarrow a_{i}\right) \rightarrow S \rightarrow S,
$$

and detaching these from (A3) gives

$$
\vdash\left(a_{i} \rightarrow a_{i}\right) \rightarrow(R \rightarrow S) \rightarrow R \rightarrow S .
$$

Detaching (I) gives

$$
\vdash(R \rightarrow S) \rightarrow R \rightarrow S .
$$

Note now that in the induction step of lemma 3.3 we did not use the fact that neither $Q$ nor $R$ there had repeated propositional variables, thus we can use the same technique to obtain

$$
\vdash(T \rightarrow R \rightarrow S) \rightarrow T \rightarrow R \rightarrow S
$$

or

$$
\vdash((R \rightarrow S) \rightarrow T) \rightarrow(R \rightarrow S) \rightarrow T) \text { etc. }
$$

until $\vdash Q \rightarrow Q$ is built up.

LEMMA 3.6

For any formula $P, \vdash P \rightarrow P$.

Proof. Let the variables of $P$ be $a_{1}, \ldots, a_{n}$, where each $a_{i}$ occurs $k_{i}$ times. Let $Q$ be $P$, where for each $i$, the $j$ th occurrence of $a_{i}$ is replaced by $a_{i j}$. All the variables of $Q$ are therefore distinct.

Now let

$$
\begin{array}{rll}
Q_{1}=\left[a_{12} / a_{11}\right] Q, & & P_{1}=Q_{1} \\
Q_{2}=\left[a_{13} / a_{12}\right] Q, & & P_{2}=\left[a_{13} / a_{12}\right] P_{1} \\
& \vdots & \\
Q_{k_{1}-1}=\left[a_{1 k_{1}} / a_{1 k_{1}-1}\right] Q, & & P_{k_{1}-1}=\left[a_{1 k_{1}} / a_{1 k_{1}-1}\right] P_{k_{1}-2} \\
Q_{k_{1}}=\left[a_{22} / a_{21}\right] Q, & & P_{k_{1}}=\left[a_{22} / a_{21}\right] P_{k_{1}-1} \\
& \vdots & \\
Q_{m}=\left[a_{n k_{n}} / a_{n k_{n}-1}\right] Q, & & P_{m}=\left[a_{n k_{n}} / a_{n k_{n}-1}\right] P_{m-1}
\end{array}
$$


where $m=\sum_{i=1}^{n}\left(k_{i}-1\right)$.

Clearly $P_{m}=P$.

By lemma 3.5 for each $k(1 \leq k \leq m)$ in $P$,

$$
\vdash Q_{k} \rightarrow Q_{k}
$$

We prove, by induction on $k, \vdash P_{k} \rightarrow P_{k}$.

The $k=1$ case is by lemma 3.5 .

Now assume $\vdash P_{k} \rightarrow P_{k}$ and detach this from lemma 3.1 to obtain

$$
\vdash\left(P_{k} \rightarrow P_{k}\right) \rightarrow P_{k} \rightarrow P_{k}
$$

Detach $Q_{k+1} \rightarrow Q_{k+1}$ from this to obtain

$$
\vdash P_{k+1} \rightarrow P_{k+1}
$$

Note that the pair of identical variables in $Q_{k+1}$ causes the corresponding pair of distinct variables in $P_{k}$ to become identical in $P_{k+1}$.

Thus we have $\vdash P_{m} \rightarrow P_{m}$ i.e. $\vdash P \rightarrow P$.

THEOREM 3.7

If $P$ is a substitution instance of $Q$ and $\vdash Q$ then $\vdash P$.

Proof. By lemma 3.6, $\vdash P \rightarrow P$. Detaching $Q$ from this gives the result.

\section{Other D-complete logics}

THEOREM 4.1

Any logic having (I), (K) and (A3) as axioms is D-complete.

Proof. All the uses of (A1) and (A2) in the work above can be performed by (A3) and $(\mathrm{K})$ instead. We give one example of this:

In the proof of lemma 3.3 we have $\vdash Q \rightarrow Q$, so by $(\mathrm{K})$ we have

$$
\vdash c \rightarrow(Q \rightarrow Q) .
$$

Detaching this from (A3) gives the result obtained previously by detaching $Q \rightarrow Q$ from (A1).

THEOREM 4.2

The logic whose axioms are (I) as well as

$$
\begin{gathered}
\left(a_{1} \rightarrow a_{2}\right) \rightarrow\left(c \rightarrow b_{1} \rightarrow b_{2}\right) \rightarrow c \rightarrow\left(a_{2} \rightarrow b_{1}\right) \rightarrow a_{1} \rightarrow b_{2} \\
\left(b_{1} \rightarrow b_{2}\right) \rightarrow\left(c \rightarrow a_{1} \rightarrow a_{2}\right) \rightarrow c \rightarrow\left(a_{2} \rightarrow b_{1}\right) \rightarrow a_{1} \rightarrow b_{2} \\
\left(c \rightarrow a_{1} \rightarrow a_{2}\right) \rightarrow\left(c \rightarrow b_{1} \rightarrow b_{2}\right) \rightarrow c \rightarrow\left(a_{2} \rightarrow b_{1}\right) \rightarrow a_{1} \rightarrow b_{2}
\end{gathered}
$$

is D-complete as is the equivalent logic having $(\mathrm{I}),(\mathrm{B}),\left(\mathrm{B}^{\prime}\right)$ and $(\mathrm{AA} 3)$.

ProOF. It can easily be checked that the above lemmas and theorem 3.7 can be proved by the above alternatives and (I), so the logic of these is $\mathbf{D}$-complete.

(AA1) can be derived from $(\mathrm{B})$ and $\left(\mathrm{B}^{\prime}\right)$ (proof: $\mathbf{B B}\left(\mathbf{B}\left(\mathbf{B}^{\prime} \mathbf{B}\right)\left(\mathbf{B B B}^{\prime}\right)\right)$ ). (B) can also be derived from (AA1) by detaching (I) twice.

(AA2) can be derived from $(\mathrm{B})$ and $\left(\mathrm{B}^{\prime}\right)$ (proof: $\left.\mathbf{B}\left(\mathbf{B}^{\prime}\left(\mathbf{B B}^{\prime}\right)\right)(\mathbf{B B}(\mathbf{B B B}))\right) .\left(\mathrm{B}^{\prime}\right)$ can also be derived from (AA2) by detaching (I) twice. 
To derive (AA3) from $(\mathrm{B})$ and $\left(\mathrm{B}^{\prime}\right)$ we require at least one axiom that has at least one propositional variable appear more than twice, such as any of $(\mathrm{W})$,

(S) $\quad(a \rightarrow b \rightarrow c) \rightarrow(a \rightarrow b) \rightarrow a \rightarrow c$ or

$\left(\mathrm{S}^{\prime}\right) \quad(a \rightarrow b) \rightarrow(a \rightarrow b \rightarrow c) \rightarrow a \rightarrow c$.

Proofs are:

$$
\begin{gathered}
\mathbf{B}(\mathbf{B W})\left\{\mathbf{B}\left(\mathbf{B}^{\prime}[\mathbf{B}(\mathbf{B B})(\mathbf{B B})]\right)\left[\mathbf{B B}\left(\mathbf{B B}^{\prime}\left(\mathbf{B B}^{\prime}\right)\right)\right]\right\} \\
\mathbf{B}\left(\mathbf{B}^{\prime}(\mathbf{B B})\right)\left(\mathbf{B S}\left(\mathbf{B}\left(\mathbf{B B}^{\prime}\right)\left(\mathbf{B B}^{\prime}\right)\right)\right) \text { and } \\
\mathbf{B}\left(\mathbf{B}^{\prime}(\mathbf{B}(\mathbf{B B})(\mathbf{B B}))\right)\left(\mathbf{B S}^{\prime}\left(\mathbf{B B}^{\prime}\right)\right)
\end{gathered}
$$

Thus:

THEOREM 4.3

The logics with axioms (B), ( $\left.\mathrm{B}^{\prime}\right),(\mathrm{I})$ and one of $(\mathrm{S}),\left(\mathrm{S}^{\prime}\right)$ or $(\mathrm{W})$ are $\mathbf{D}$-complete.

Logics such as these look simpler than $M$ however they are stronger.

THEOREM 4.4

The logic $M$ is strictly weaker than $\mathbf{B B}^{\prime} \mathbf{I S}, \mathbf{B B}^{\prime} \mathbf{I} \mathbf{W}, \mathbf{B B}^{\prime} \mathbf{I} \mathbf{S}^{\prime}$ or $\mathbf{B B}^{\prime} \mathbf{I}(\mathrm{AA} 3)$ logic.

Proof. Theorem 4.2 and the discussion below it showed that $\mathbf{B B}^{\prime} \mathbf{I}(\mathrm{AA} 3)$ is a subsystem of each of $\mathbf{B B}^{\prime} \mathbf{I S}, \mathbf{B B}^{\prime} \mathbf{I} \mathbf{S}^{\prime}$ and $\mathbf{B B}^{\prime} \mathbf{I} \mathbf{W}$. The axioms of $M$ are substitution instances of (I), (AA1), (AA2) and (AA3), so by theorem 4.2, $M$ is a subsystem of, or is, $\mathbf{B B}^{\prime} \mathbf{I}(\mathrm{AA} 3)$.

It is easy to show, by induction on the length of proof, that all theorems of $M$ are of the form

$$
\vdash P_{1} \rightarrow P_{2} \rightarrow \cdots P_{n} \rightarrow Q \rightarrow Q
$$

for some $n \geq 0$. Thus (B) and $\left(\mathrm{B}^{\prime}\right)$ cannot be derived so $M$ is weaker than the 4 logics above.

THEOREM 4.5

The logic with (B), ( $\left.\mathrm{B}^{\prime}\right),(\mathrm{I})$ and

$$
\left(\mathrm{I}^{\prime}\right) \quad(a \rightarrow a \rightarrow b) \rightarrow a \rightarrow a \rightarrow b
$$

is $\mathbf{D}$-complete.

Proof. The proof $\mathbf{B}\left[\mathbf{B}^{\prime}\{\mathbf{B}(\mathbf{B B})(\mathbf{B B})\}\right]\left[\mathbf{B B}\left(\mathbf{B B}^{\prime}\left(\mathbf{B B}^{\prime}\right)\right)\right]$ gives

$$
\begin{gathered}
\left(\mathrm{AA}^{\prime}\right) \quad \vdash_{\mathrm{BB}^{\prime}}\left(c_{1} \rightarrow a_{1} \rightarrow a_{2}\right) \rightarrow\left(c_{2} \rightarrow b_{1} \rightarrow b_{2}\right) \rightarrow c_{2} \rightarrow c_{1} \rightarrow \\
\left(a_{2} \rightarrow b_{1}\right) \rightarrow a_{1} \rightarrow b_{2} .
\end{gathered}
$$

$\mathbf{B}\left(\mathbf{B I}^{\prime}\right)\left(\mathrm{AA} 3^{\prime}\right)$ then gives

$$
\begin{aligned}
\left(\mathrm{AA}^{\prime \prime}\right) \quad \vdash_{\mathrm{BB}^{\prime} \mathbf{I}^{\prime}}\left(c \rightarrow a_{1} \rightarrow a_{2}\right) \rightarrow & \left(c \rightarrow b_{1} \rightarrow b_{2}\right) \rightarrow c \rightarrow c \rightarrow\left(a_{2} \rightarrow b_{1}\right) \\
& \rightarrow a_{1} \rightarrow b_{2}
\end{aligned}
$$


using which the lemmas and theorem 3.7 can be proved as with (A3).

As $\left(\mathrm{AA}^{\prime}\right)$, and so $\left(\mathrm{AA}^{\prime \prime}\right)$, is a $\mathbf{B B}^{\prime} \mathbf{I}$ theorem, it follows that $\mathbf{B B}^{\prime} \mathbf{I}$ and $\mathbf{B B}^{\prime} \mathbf{I}\left(\mathrm{AA}^{\prime \prime}\right)$ are equivalent logics. In theorems of condensed $\mathbf{B B}^{\prime} \mathbf{I}$ (or $\mathbf{B C I}$ ) logic propositional variables must appear exactly twice each, thus $\left(\mathrm{AA} 3^{\prime \prime}\right)$ is not provable in condensed $\mathbf{B B}^{\prime} \mathbf{I}$ logic. It therefore follows that condensed versions of equivalent logics may be inequivalent.

$M$ is not the weakest $\mathbf{D}$-complete logic, this we show below.

THEOREM 4.6

The logic $M^{*}$ with (I) and

$$
\begin{aligned}
& (\mathrm{A} 1)^{*} \quad(a \rightarrow a) \rightarrow((c \rightarrow c) \rightarrow b \rightarrow b) \rightarrow(c \rightarrow c) \rightarrow(a \rightarrow b) \rightarrow a \rightarrow b \\
& (\mathrm{~A} 2)^{*} \quad(b \rightarrow b) \rightarrow((c \rightarrow c) \rightarrow a \rightarrow a) \rightarrow(c \rightarrow c) \rightarrow(a \rightarrow b) \rightarrow a \rightarrow b \\
& (\mathrm{~A} 3)^{*} \quad((c \rightarrow c) \rightarrow a \rightarrow a) \rightarrow((c \rightarrow c) \rightarrow b \rightarrow b) \rightarrow(c \rightarrow c) \rightarrow(a \rightarrow b) \rightarrow a \rightarrow b
\end{aligned}
$$

is $\mathbf{D}$-complete and strictly weaker than $M$.

Proof. All the lemmas prior to it and theorem 3.7 can be proved as before.

As $M$ is $\mathbf{D}$-complete and (A1)*, (A2)* and (A3)* are substitution instances of (A1), (A2) and (A3) respectively, they are theorems of $M$.

It can easily be shown that all theorems of $M^{*}$ have the property that all propositional variables occur an even number of times in any theorem. This does not hold for (A3), so (A3) is not a theorem of $M^{*}$.

Thus $M^{*}$ is strictly weaker than $M$.

An infinite set of independent $\mathbf{D}$-complete logics can be obtained by considering systems $M_{n}$ with as axioms (I), (A1), (A2) and

$$
(\mathrm{A} 3 \mathrm{n}) \quad(c \rightarrow a \rightarrow a) \rightarrow(c \rightarrow b \rightarrow b) \rightarrow c \rightarrow \ldots \rightarrow c \rightarrow(a \rightarrow b) \rightarrow(a \rightarrow b)
$$

where $c \rightarrow \ldots \rightarrow c$ contain $n \geq 1 c$ 's. (Note that $M_{1} \equiv M$.)

\section{THEOREM 4.7}

The systems $M_{n}$ are mutually independent and weaker than $\mathbf{B B}^{\prime} \mathbf{I W}$.

Proof. We first show that (A3m) is not derivable in $M_{n}$ where $n \geq 3$ and $m\langle n$.

Consider the matrix

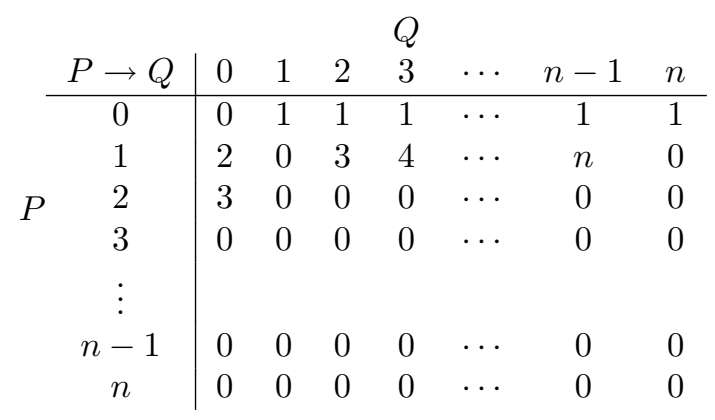

where 0 is the only designated value.

Clearly any formula of the form $P \rightarrow P$ (including (I)) has value 0 .

Thus (A1) and (A2) can be evaluated as 


$$
\begin{array}{l|rrrrr}
c & 0 & \rightarrow & (c \rightarrow 0) & \rightarrow & (c \rightarrow 0) \\
\hline & & 0 & & 0
\end{array}
$$

and $(\mathrm{A} 3 m)$ as

\begin{tabular}{c|cccccl}
$c$ & $(c \rightarrow 0)$ & $\rightarrow$ & $(c \rightarrow 0)$ & $\rightarrow$ & $c \rightarrow \cdots \rightarrow c \rightarrow 0$ & \\
\cline { 1 - 5 } & 2 & 3 & 2 & 0 & $m+1$ & if $m\langle n$ \\
1 & 2 & 0 & 2 & 3 & 0 & if $m=n$ \\
2 & 3 & 0 & 3 & 0 & 3 & if $m=1$ \\
2 & 3 & 0 & 3 & 0 & 0 & if $m>1$ \\
other & 0 & 0 & 0 & 0 & 0 &
\end{tabular}

The $n$-valued matrix for $\rightarrow$ validates all theorems of $M_{n}$ if $n>2$ but not all of those of $M_{m}$ if $m\langle n$.

We now show that (A3m) is not derivable in $M_{n}$ where $\left.m\right\rangle n \geq 2$.

Consider the matrix with designated value 0 .

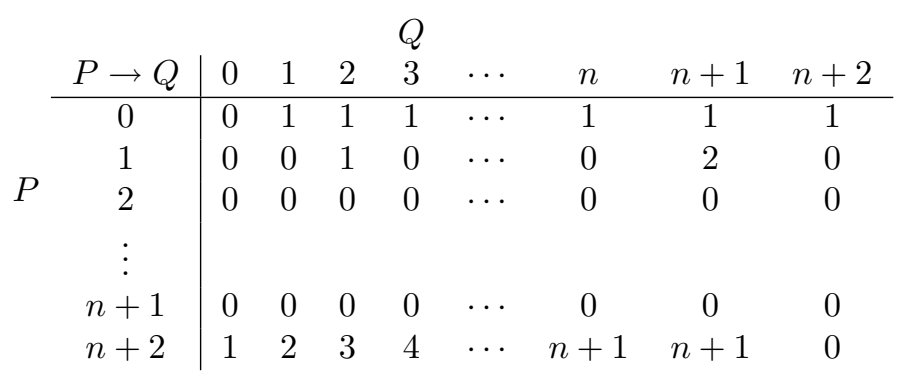

(A1) and (A2) can be evaluated as before and (A3m) as

\begin{tabular}{c|cccccc}
$c$ & $(c \rightarrow 0)$ & $\rightarrow$ & $(c \rightarrow 0)$ & $\rightarrow$ & $c \rightarrow \cdots \rightarrow c \rightarrow 0$ & \\
\hline$n+2$ & 1 & 0 & 1 & 0 & $n$ & if $m=n$ \\
$n+2$ & 1 & 1 & 1 & 2 & $n+1$ & if $m>n$ \\
other & 0 & 0 & 0 & 0 & 0 &
\end{tabular}

When $m>n=1$, consider the matrix with designated value 0 .

\begin{tabular}{|c|c|c|c|c|c|}
\hline \multirow{2}{*}{\multicolumn{2}{|c|}{$P \rightarrow Q$}} & & \multicolumn{3}{|c|}{$Q$} \\
\hline & & 0 & 1 & 2 & 3 \\
\hline & 0 & 0 & 1 & 1 & 1 \\
\hline & 1 & 0 & 0 & 2 & 0 \\
\hline & 2 & 0 & 0 & 0 & 0 \\
\hline & 3 & 1 & 2 & 2 & 0 \\
\hline
\end{tabular}

(A1) and (A2) can be evaluated as before and (A3m) as

\begin{tabular}{c|cccccc}
$c$ & $(c \rightarrow 0)$ & $\rightarrow$ & $(c \rightarrow 0)$ & $\rightarrow$ & $c \rightarrow \cdots \rightarrow c \rightarrow 0$ & \\
\cline { 1 - 5 } 3 & 1 & 0 & 1 & 0 & 1 & $m=n$ \\
3 & 1 & 2 & 1 & 2 & 2 & $m>n$ \\
other & 0 & 0 & 0 & 0 & 0 &
\end{tabular}

Thus all theorems of $M_{n}$ are validated for $n \geq 1$ but not all of $M_{m}$ for $m>n$.

Finally, all the theorems of $M_{2}$ are $\mathbf{B B}^{\prime} \mathbf{I}$ theorems and (A3)(=A31), a theorem of $M_{1}$, is not. 
We therefore have that each system $M_{n}$ has a theorem not provable in $M_{m}$ if $m \neq n$, so all the systems are independent.

We show that each system $M_{n}$ is no stronger than $\mathbf{B B}^{\prime} \mathbf{I W}$ by stating a proof in $\mathbf{B B}^{\prime} \mathbf{I} \mathbf{W}$ of a formula of which (A3n) is a substitution instance. The $\mathbf{D}$-completeness of $\mathbf{B B}^{\prime} \mathbf{I} \mathbf{W}$ means that (A3n) is also derivable (by methods such as the ones in this paper). The proof for $n>2$ is:

$$
\mathbf{B}\left(\mathbf{B}^{\prime}\left(\mathbf{B B}^{n}\right)\right)\left\{\mathbf{B B}\left(\mathbf{B B}^{\prime}\left[\mathbf{B}\left(\mathbf{B}^{n-1} \mathbf{B}^{\prime}\right)\left(\mathbf{W}\left[\mathbf{B}\left(\mathbf{B}^{\prime}\left(\mathbf{B B}^{n-2}\right)\right)\left(\mathbf{B B} U_{n-2}\right)\right]\right)\right]\right)\right\},
$$

where $U_{1}=\mathbf{B}^{\prime}, U_{i+1}=\mathbf{B B}^{\prime}\left(\mathbf{W}\left(\mathbf{B}\left\{\mathbf{B}^{\prime}\left(\mathbf{B B}^{i}\right)\right\}\left(\mathbf{B B} U_{i}\right)\right)\right), \mathbf{B}^{1}=\mathbf{B}, \mathbf{B}^{i+1}=\mathbf{B B B}^{i}$.

The fact that $M_{n}$ for $n>2$ is strictly weaker than $\mathbf{B B}^{\prime} \mathbf{I W}$ follows as in the proof of theorem 4.4. As all the axioms of $M_{2}$ are $\mathbf{B B}^{\prime} \mathbf{I}$ theorems, $M_{2}$ is strictly weaker than $\mathbf{B B}^{\prime} \mathbf{I W}$. This result for $M_{1}$ appears in theorem 4.4 .

\section{5 'Completing' by substitution}

The logic $\mathbf{I}(\mathrm{AA} 1)(\mathrm{AA} 2)\left(\mathrm{AA}^{\prime}\right)$ is equivalent to $\mathbf{B B}^{\prime} \mathbf{I}$ logic; neither is D-complete. However, as was shown in the proof of theorem 4.5, one simple (variable for variable) substitution in $\left(\mathrm{AA} 3^{\prime}\right)$ (giving $\left(\mathrm{AA} 3^{\prime \prime}\right)$ ) converts the logic into a $\mathbf{D}$-complete one. To convert $\mathbf{B B}^{\prime} \mathbf{I}$ logic into a $\mathbf{D}$-complete logic, a single more complex substitution instance of $(\mathrm{B})$ must be added to $(\mathrm{B}),\left(\mathrm{B}^{\prime}\right)$ and $(\mathrm{I})$. This allows the proof of $\left(\mathrm{AA} 3^{\prime}\right)$ in the proof of theorem 4.5 to be converted into the proof of the other $\mathbf{B B}^{\prime} \mathbf{I}$ theorem quoted there.

However, we prove below that I logic requires an infinite number of substitution instances of its axiom to convert it into a $\mathbf{D}$-complete logic and conjecture that the same is true for the logics $\mathbf{B}, \mathbf{B}^{\prime}, \mathbf{B B}^{\prime}, \mathbf{B I}$ and $\mathbf{B}^{\prime} \mathbf{I}$.

We now look at theorems that can be generated using Rule $\mathbf{D}$ from substitution instances of Axiom (I). For this we need to define unification.

DEFINITION 5.1

$\mathrm{U}\left(P_{1}, P_{2}\right)$ is the unification of $P_{1}$ and $P_{2}$. This is the shortest formula $\sigma_{1}\left(P_{1}\right)$ such that for some $\sigma_{2}, \sigma_{1}\left(P_{1}\right)=\sigma_{2}\left(P_{2}\right)$ and of those one whose number of distinct propositional variables is maximal.

In other words $\mathrm{U}\left(P_{1}, P_{2}\right)=\sigma_{1}\left(P_{1}\right)$ the result of detaching $P_{2}$ from $P_{1} \rightarrow P_{1}$.

We define

$$
\mathrm{U}\left(P_{1}, \ldots, P_{n}\right)=\mathrm{U}\left(\mathrm{U}\left(P_{1}, \ldots, P_{n-1}\right), P_{n}\right) .
$$

We note that

$$
\begin{gathered}
\mathrm{U}\left(\mathrm{U}\left(P_{11}, \ldots, P_{1 n_{1}}\right), \ldots, \mathrm{U}\left(P_{k 1}, \ldots, P_{k n_{k}}\right)\right)= \\
\mathrm{U}\left(P_{11}, \ldots, P_{1 n_{1}}, \ldots, P_{k 1}, \ldots, P_{k n_{k}}\right) .
\end{gathered}
$$

LEMMA 5.2

If $Q \rightarrow Q$ is derived using only Rule $\mathbf{D}$ from $P_{1} \rightarrow P_{1}, \ldots, P_{n} \rightarrow P_{n}$ as axioms then each subterm of $Q \rightarrow Q$ is of the form $\mathrm{U}\left(R_{1}, \ldots, R_{k}\right)$ where $R_{1}, \ldots, R_{k}$ are (sub)terms of $P_{1}, P_{2}, \ldots, P_{n}$.

Proof. The result clearly holds for any axiom (with $\left.\mathrm{U}\left(P_{i}\right) \equiv P_{i}\right)$.

If $Q \rightarrow Q$ comes from detaching $S \rightarrow S$ from $T \rightarrow T$ then

(i) if $T$ is a propositional variable 


$$
Q=S
$$

and the result holds by the induction hypothesis, and

(ii) if $T \equiv T_{1} \rightarrow T_{2}$ then

$$
Q=\mathrm{U}\left(T_{1}, T_{2}, S\right)
$$

By the induction hypothesis

$$
\begin{aligned}
& T_{1}=\mathrm{U}\left(R_{1}, \ldots, R_{k}\right) \\
& T_{2}=\mathrm{U}\left(R_{i}, \ldots, R_{j}\right) \quad \text { and } \\
& S=\mathrm{U}\left(R_{p}, \ldots, R_{q}\right)
\end{aligned}
$$

where $R_{1}, \ldots, R_{k}, R_{i}, \ldots, R_{j}, R_{p}, \ldots, R_{q}$ are (sub)terms of $P_{1}, \ldots, P_{n}$.

Therefore $Q=\mathrm{U}\left(R_{1}, \ldots, R_{k}, R_{i}, \ldots, R_{j}, R_{p}, \ldots, R_{q}\right)$ as required.

LEMMA 5.3

The number of elements generated by unification from $P_{1}, \ldots, P_{n}, \# \mathrm{UE}\left(P_{1}, \ldots, P_{n}\right) \leq$ $2^{n}-1$.

Proof. The number of unifications $\mathrm{U}\left(P_{i}, P_{j}\right)$ for $i \neq j$ is clearly at most $n(n-1) / 2$.

The number of unifications, for $i, j, k$ different,

$$
\begin{aligned}
\mathrm{U}\left(\mathrm{U}\left(P_{i}, P_{j}\right), P_{k}\right) & =\mathrm{U}\left(\mathrm{U}\left(P_{i}, P_{j}\right), \mathrm{U}\left(P_{i}, P_{k}\right)\right) \\
& =\mathrm{U}\left(\mathrm{U}\left(P_{i}, P_{j}\right), \mathrm{U}\left(P_{j}, P_{k}\right)\right) \\
& =\mathrm{U}\left(\mathrm{U}\left(P_{k}, P_{i}\right), \mathrm{U}\left(P_{j}, P_{k}\right)\right)
\end{aligned}
$$

is at the most $n(n-1)(n-2) /(1 \cdot 2 \cdot 3)$.

So

$$
\begin{aligned}
\# \mathrm{UE}\left(P_{1}, \ldots, P_{n}\right) \leq & n+\frac{n(n-1)}{1 \cdot 2}+\frac{n(n-1)(n-2)}{1 \cdot 2 \cdot 3}+\cdots+ \\
& \frac{n(n-1)}{1 \cdot 2}+n+1 \\
\leq & 2^{n}-1
\end{aligned}
$$

\section{THEOREM 5.4}

Only a finite number of theorems can be derived using Rule $\mathbf{D}$ only from axioms of the form $P_{1} \rightarrow P_{1}, \ldots, P_{n} \rightarrow P_{n}$.

Proof. By lemma 5.2 any theorem derived from such axioms using Rule $\mathbf{D}$ is of the form $Q \rightarrow Q$ where $Q$ is a unification of (sub)terms of $P_{1}, \ldots, P_{n}$. The number of such (sub)terms is finite so by lemma 5.3 the number of terms $Q$ that can be generated by unification is also finite.

THEOREM 5.5

To transform condensed I-logic into I-logic an infinite number of substitution instances of the axiom $a \rightarrow a$ are required.

Proof. By theorem 5.4.

Note that there are also logics where substitution instances of their axioms give no new theorems other than those axioms themselves. An example is the logic with the axiom

$$
(a \rightarrow a) \rightarrow(a \rightarrow a) \rightarrow a \rightarrow a
$$




\section{References}

[1] Bunder, M. W. A simplified version of condensed detachment. Journal of Logic, Language and Information, to appear.

[2] Hindley, J. R. Basic Simple Type Theory. Cambridge University Press, 199?, to appear.

[3] Hindley, J. R. and Meredith, D. Principal type-schemes and condensed detachment. J. Symbolic Logic 55:90-105, 1990.

[4] Kalman, J. Condensed detachment as a rule of inference. Studia Logica 42:443-451, 1983.

[5] Lemmon, E. J., Meredith, C. A., Meredith, D., Prior, A. N., Thomas, I. Calculi of pure strict implication. Philosophical Logic Reidel, Dordrecht (1969) pp. 215-250. (Original date: 1957).

[6] Meyer, R. K. and Bunder, M. W. Condensed detachment and combinators. Tech. Report TRARP-8188, Research School of Social Sciences, Aus. Nat. Univ. Canberra, Australia, 1988.

[7] Mints, G. E. and Tammet, T. Condensed detachment is complete for relevance logic: a computeraided proof. Journal of Automated Reasoning 7:587-596, 1991.

[8] Robinson, J. A., A machine-oriented logic based on the resolution principle. Journal of the Association for Computing Machinery 12:23-41, 1965.

Received 17 May 1995. Revised 20 October 1995 
